\title{
RATES AND PATTERNS OF MOLECULAR EVOLUTION IN MARINE ANIMALS FOLLOWING THE ISTHMIAN EMERGENCE
}

COLLINS, Timothy, Department of Biology, Kraus Natural Sciences Bldg., University of Michigan, Ann Arbor, Michigan 48109-1048 U.S.A.

The marine vicariant event resulting from the Pliocene emergence of the Central American Isthmus presents a unique opportunity for calibrating rates of molecular evolution. The synchronous fragmentation of the ranges of previously widespread taxa into Western Atlantic and Eastern Pacific components (geminates) enables one to make comparisons of rates among higher taxa on the same time scale and to evaluate the regularity of rates of molecular evolution among all species sampled. Other advantages of this approach are that the time scale (approximately 3 $\mathrm{Ma}$ ) is one of particular interest for evolutionary biologists concerned with speciation and one that minimizes the ambiguities associated with augmentation of divergence values to account for multiple hits at a site. The divergence values derived for geminate pairs are independent, allowing statistical evaluation of variance in rates.

The current popularity of the relative rates test as the final arbiter of questions regarding rates and rate variation is primarily a matter of convenience and not a reflection of methodological superiority. A review of the commonly used techniques for calibrating rates of molecular evolution shows that each approach has limitations. Temporally based calibrations of rates are necessary complements to time-independent comparisons.

Interpretation of transisthmian molecular comparisons in the literature have in many cases been unduly influenced and confused by molecular clock assumptions and the restriction of studies to single higher-level taxa. Analysis of the apparently contradictory published data as well as new results from sequence comparisons of fishes, urchins and snails suggests a synthesis: taxon specific rates of molecular evolution, with reduced variance within taxonomic groups and great variance among all groups sampled. 